\title{
Ranking Participants in Generalized Tournaments
}

\author{
Giora Slutzki* and Oscar Volij ${ }^{\dagger}$
}

September 8, 2004

\begin{abstract}
Consider the problem of ranking social alternatives based on the number of voters that prefer one alternative to the other. Or consider the problem of ranking chess players by their past performance. A wide variety of ranking methods have been proposed to deal with these problems. Using six independent axioms, we characterize the fair-bets ranking method proposed by Daniels [4] and Moon and Pullman [14].
\end{abstract}

Keywords: Tournaments, ranking and scoring methods, Perron-Frobenius theorem, Birkhoff's theorem.

\section{Introduction}

A tournament is a pair $\langle N, A\rangle$ where $N$ is a finite set of at least two elements, which we will call players, and $A$ is a zero-one matrix, such that its diagonal entries are zeroes and for all $i, j \in N, i \neq j, a_{i j}+a_{j i}=1$. The interpretation is that each pair of players in $N$ played each other once, and $a_{i j}=1$ if, and only if, $i$ beat $j$. Tournaments arise naturally in many contexts. Clearly, sports events are the first ones that come to mind but they also arise

*Department of Computer Science, Iowa State University, Ames, IA 50011, USA. Email: slutzki@cs.iastate.edu.

${ }^{\dagger}$ Department of Economics, Iowa State University, Ames, IA 50011, USA.

Email: oscar@volij.co.il. http://volij.co.il. 
in the context of social choice [Arrow (1963), Sen (1970), Moulin (1988)], and in statistics [David (1987), David (1988)]. For example, if $N$ represents a set of social alternatives, then $A$ can represent the majority rule binary relation: $a_{i j}=1$ if there is a majority of voters that prefer $i$ to $j$, and $a_{i j}=0$ otherwise. See Moon (1968) and Laslier (1997) for a comprehensive treatment of tournaments including more applications and examples.

Researchers have been interested in both cardinal and ordinal aspects of tournaments, as well as of more general structures. Scoring methods are intended to capture the cardinal aspect by assigning a numerical score to each participant in a tournament. Such a score presumably quantifies how good each of the players is relative to other players. Ranking methods, on the other hand, intend to capture the ordinal aspect of a tournament by assigning a ranking of the players. By contrast to a scoring method, a ranking method does not convey any quantitative information as to how much better a player is relative to another, just the mere fact that one player is better than another.

Much work has been done on scoring systems and ranking methods. Early references include Zermelo (1929), Wei (1952), Kendall (1955), Daniels (1969), Moon and Pullman (1970). Since this pioneering work, the literature on ranking methods has expanded significantly, both in terms of quantity and scope. ${ }^{1}$

Rubinstein (1980) characterized the ranking method that ranks players in a tournament according to the number of their respective victories, also known as Copeland scores. ${ }^{2}$ This method, which was called the points system, treats all victories equally. A nice feature of Rubinstein's characterization is that it is in terms of a fixed, rather than variable, set of players.

Daniels (1969) and Moon and Pullman (1970) proposed another method for ranking players in a tournament, which we call the fair-bets method, according to which beating highly ranked players is more valuable than beating low ranked players. ${ }^{3}$ In this paper we look for axioms that characterize the fair-bets ranking method. In order to do so we

\footnotetext{
${ }^{1}$ See for instance Kano and Sakamoto (1985), David (1987), Keener (1993), Levchenkov (1995), van den Brink and Gilles (2000), Herings, van der Laan, and Talman (2004), Palacios-Huerta and Volij (2004), Conner and Grant (2000), as well as the comprehensive texts of David (1988) and Laslier (1997).

${ }^{2}$ Henriet (1985) and van den Brink and Gilles (2000) provided, later on, alternative characterizations. See also Bouyssou (1992)

${ }^{3}$ The method was originally defined for irreducible tournaments, but it can be extended in a straightforward way to a method where the tournament is not irreducible.
} 
need to extend the class of problems over which the methods are defined to more general kinds of tournaments. Specifically, we allow for problems in which different pairs may have different number of encounters, possibly none. Further, for reasons that have to do with the axioms we use, we cannot restrict attention to a fixed set of players, but must also consider its subsets. We now turn to the description of our axioms.

Rubinstein (1980) required a method to exhibit positive responsiveness with respect to the beating relation. Specifically, the requirement is that switching a player's loss to a win cannot have a negative effect on that player's rank, and must have a positive effect if he was tied with some other player. Although the fair-bets method satisfies this property (see Laslier (1997), pp. 59-62 or Levchenkov (1992)), it does not seem to help in characterizing the method. Instead, we use an axiom, called negative responsiveness to losses, which requires that under certain circumstances, multiplying a player's losses by a constant greater than one has a negative impact on his rank. Note that this operation takes us out of the class of tournaments, i.e., the class of tournaments is not closed under multiplication of a player's losses by a constant. This technical reason forces us to enlarge the class of problems under consideration.

In a previous paper, Slutzki and Volij (2004) characterized the fair-bets scoring method for the class of irreducible problems. One of the crucial axioms used there states that all players receive the same score in a particular class of simple problems, namely, the class of strongly balanced problems. ${ }^{4}$ In this paper we replace this arguably strong axiom by the more appealing property of anonymity. This replacement, however, comes at the cost of a further enlargement of the class of problems that need to be considered. For the full power of anonymity to be utilized, we have to consider some reducible problems, namely those in which there are several unconnected "leagues." This extension allows us to apply Birkhoff's theorem on doubly stochastic matrices which, together with anonymity, enables us to conclude that in strongly balanced problems all players must be equally ranked.

Another axiom we use is separability. This axiom allows us to rank players in a given league independently of the outcomes in other leagues. Specifically, separability allows us to rank the players in a given league by regarding it as a separate ranking problem. Since a league consists of a particular subset of players and its corresponding bilateral outcomes,

\footnotetext{
${ }^{4} \mathrm{~A}$ problem is strongly balanced if all the players have the same number of wins and the same number of losses.
} 
this axiom forces us to consider not only a given set of players but also its subsets.

Another crucial axiom is quasi-flatness preservation which says that if in two ranking problems (with the same set of players), any two players are either equally ranked or incomparable, the same should be true if we merge the two problems into a single one.

Finally, the last two axioms deal with the fact that the class of problems we consider includes reducible ones. Since in some problems there might not be enough grounds for comparison between two players, one should not expect a ranking method to deliver a complete order of the participants for all problems. The axiom of quasi-completeness allows the method to "declare" two players as incomparable precisely when they have never played against each other, and there is no chain of comparisons that leads from one of the players to the other. The axiom of dominance, on the other hand, requires that the ranking of the participants respects the ranking of the leagues induced by the problem. In other words, all players in a league should rank above all players in a lower league.

The paper is organized as follows. Section 2 presents the model, section 3 introduces the ranking methods that are the focus of the paper, section 4 offers the characterization of the fair-bets method and section 5 shows the independence of the axioms.

\section{The Model}

Let $\mathbb{N}=\{1,2, \ldots\}$ be the set of potential players. A (ranking) problem is a pair $\langle N, A\rangle$ where $N \subseteq \mathbb{N}$ is a finite set of players and $A$ is a $|N| \times|N|$ non-negative integer matrix whose main diagonal consists of zeroes. For any $i, j \in N$, the entry $a_{i j}$ represents the number of times player $i$ beat player $j$. For $i \in N$, we denote by $a_{i *}=\sum_{j \in N} a_{i j}$ and $a_{* i}=\sum_{j \in N} a_{j i}$ the total number of victories and the total number of losses, respectively, of player $i$. Also, we let $C_{A}=\operatorname{diag}\left\{a_{* i}\right\}_{i \in N}$ denote the diagonal matrix whose $i$-th main diagonal entry is player $i$ 's total number of losses. We denote by $\mathcal{A}$ the set of all problems.

Given a ranking problem $\langle N, A\rangle$, we say that $i$ beat $j$ if $a_{i j}>0$. We say that $i$ beat $j$ indirectly, denoted $i \rightarrow j$, if there is a finite sequence $i_{0}, i_{1}, \ldots, i_{T}$, with $i_{0}=i$ and $i_{T}=j$, such that $\prod_{t=0}^{T-1} a_{i_{t} i_{t+1}}>0$. Note that for $T=0, \prod_{t=0}^{T-1} a_{i_{t} i_{t+1}}=1$ so that " $\rightarrow$ " is a reflexive relation. Also note that " $\rightarrow$ " is the transitive and reflexive closure of the "beat" relation. We say that player $i$ belongs to the same league as player $j$, denoted $i \leftrightarrow j$, if $i \rightarrow j$ and $j \rightarrow i$. It can be checked that $\leftrightarrow$ is an equivalence relation and therefore it partitions 
the set $N$ of players into equivalence classes. We call these equivalence classes leagues, and denote the league that contains player $i$ by $[i]$. If the problem $\langle N, A\rangle$ consists of only one league, namely if $N$ constitutes a league, then we say that the problem $\langle N, A\rangle$ as well as the matrix $A$ are irreducible. We denote by $\mathcal{A}_{I}$ the set of all irreducible problems. Define the following relation between leagues. Given two players $i, j \in N$, we say that $i$ 's league is higher than $j$ 's league, denoted by $[i] \mapsto[j]$, if and only if $i \rightarrow j$ and not $j \rightarrow i$. This induces an irreflexive and transitive relation on the leagues. Two distinct leagues are incomparable if neither is higher than the other. For all $i \in N$, the sub-problem of $[i]$ is defined to be the problem $R_{[i]}=\left\langle[i], A_{[i]}\right\rangle$, where $A_{[i]}$ is the sub matrix of $A$ restricted to the players in $[i]$.

A matrix $A$ is said to be balanced, if for all $i, a_{i *}=a_{* i}$. It is strongly balanced if in addition there is a constant $k>0$ such that for all $i, a_{* i}=k$. We say that a problem $R=\langle N, A\rangle \in \mathcal{A}$ is (strongly) balanced, if $A$ is (strongly) balanced, and that league $[i]$ is (strongly) balanced if the matrix $A_{[i]}$ is (strongly) balanced.

We end this section with the following lemma, which states a useful relationship between the balancedness of a problem and the balancedness of its leagues. The proof is in the appendix.

Lemma 1 Let $R=\langle N, A\rangle$ be a problem. $R$ is balanced if and only if its leagues are incomparable and each one is balanced.

\section{Ranking Methods}

Given a problem $\langle N, A\rangle$, we are interested in ranking the players in $N$ based on the information contained in $A$. A ranking of $N$ is a binary relation on $N$ that is reflexive and transitive. We denote the set of all rankings of $N$ by $\mathcal{R}_{N}$. Given a ranking $\succeq$ of $N$, we define the following associated relations:

- $i$ is ranked above $j$, denoted $i \succ j$, if $i \succeq j$ but not $j \succeq i$,

- $i$ and $j$ are equally ranked, denoted $i \sim j$, if $i \succeq j$ and $j \succeq i$, 
- $i$ and $j$ are incomparable, denoted $i \perp j$, if neither $i \succeq j$ nor $j \succeq i$.

A ranking $\succeq$ of $N$ is quasi-flat if for all pairs of players $i, j \in N$, either $i \sim j$ or $i \perp j$. It is flat if for all pairs of players $i, j \in N, i \sim j$.

A (ranking) method is a function $\succeq: \mathcal{A} \rightarrow \cup_{N \subseteq N} \mathcal{R}_{N}$ that associates with each problem $R=\langle N, A\rangle$ a ranking, $\succeq_{R}$, of $N$.

We now introduce two examples of methods, defined only on the class of irreducible problems $\mathcal{A}_{I}$. We will see later that there is a natural way to extend methods defined on $\mathcal{A}_{I}$ to the whole class of problems $\mathcal{A}$.

Example 1 The fair-bets method.

Let $R=\langle N, A\rangle$ be an irreducible problem. Recall that $C_{A}=\operatorname{diag}\left\{a_{* i}\right\}_{i \in N}$. Since $A$ is an irreducible matrix, so is $C_{A}^{-1} A$. By the Perron-Frobenius theorem ${ }^{5}, C_{A}^{-1} A$ has a unique (up to a positive scalar multiplication) positive eigenvector $v=\left(v_{i}\right)_{i \in N}$. The components of this eigenvector satisfy the following system of homogeneous linear equations:

$$
\sum_{j \in N} a_{i j} v_{j}=\sum_{j \in N} a_{j i} v_{i} \quad i \in N .
$$

Call the entries of this eigenvector the fair-bets scores of $R$. We can use these fair-bets scores to rank the players in $R$. Formally, the fair-bets method on the class of irreducible problems is the function $\succeq^{f b}: \mathcal{A}_{I} \rightarrow \cup_{N \subseteq N} \mathcal{R}_{N}$ such that for all $R=\langle N, A\rangle \in \mathcal{A}_{I}$,

$$
i \succeq_{R}^{f b} j \quad \text { if and only if } v_{i} \geq v_{j}
$$

where $\left(v_{k}\right)_{k \in N}$ is a positive eigenvector of $C_{A}^{-1} A$.

The idea behind the fair-bets method for irreducible problems, as defined in equation (1), can be illustrated in terms of systems of bets. A list of non-negative numbers $v=$ $\left(v_{1}, \ldots, v_{n}\right)$ may be viewed as a system of bets in the following sense. A bet on $i$ is a contract whereby the bettor gets $\$ v_{j}$ each time player $i$ beats player $j$ and he pays out $\$ v_{i}$ each time player $i$ loses regardless of $i$ 's opponent's identity. The bet on $i$ is fair if the total

\footnotetext{
${ }^{5}$ The reader is referred to Minc (1988) for an excellent treatment of the theory of non-negative matrices.
} 
payment due to $i$ 's losses equals the total revenue obtained from $i$ 's victories. A system $v=\left(v_{1}, \ldots, v_{n}\right)$ of bets is fair if the associated bets on each player are all fair. Given a fair system of bets, $v=\left(v_{1}, \ldots, v_{n}\right)$, we can rank the players according to their respective $v_{i}$ 's. It turns out that given an irreducible problem, all the fair systems of bets induce the same ranking on the set of players. Moreover, the fair-bets method ranks the players precisely according to this unique ranking.

Example 2 The dual fair-bets method.

Define the dual fair-bets method $\succeq^{d f b}: \mathcal{A}_{I} \rightarrow \cup_{N \subseteq N} \mathcal{R}_{N}$ as follows. For all $R=\langle N, A\rangle \in \mathcal{A}$,

$$
i \succeq_{R}^{d f b} j \text { if and only if } j \succeq_{R^{T}}^{f b} i
$$

where $R^{T}=\left\langle N, A^{T}\right\rangle$ is the "transposed" problem, where victories are treated as losses and losses are treated as victories.

To illustrate the above methods, let us consider the four-player tournament $R=\langle N, A\rangle$ where $N=\{1,2,3,4\}$ and $A$ is given by

$$
\left(\begin{array}{llll}
0 & 1 & 1 & 0 \\
0 & 0 & 1 & 1 \\
0 & 0 & 0 & 1 \\
1 & 0 & 0 & 0
\end{array}\right) .
$$

The points system would rank participants 1 and 2 equally and above participants 3 and 4, who would also be ranked equally. This is because both players 1 and 2 have two wins while players 3 and 4 have only one win. Noting that $\left(v_{1}, v_{2}, v_{3}, v_{4}\right)=(4,3,1,2)$ constitutes a fair system of bets, namely it solves

$$
\sum_{j \in N} a_{i j} v_{j}=\sum_{j \in N} a_{j i} v_{i} \quad i \in N
$$

we conclude that the fair-bets method ranks the players as follows: $1 \succeq_{R}^{f b} 2 \succeq_{R}^{f b} 4 \succeq_{R}^{f b} 3$. On the other hand, since $\left(v_{1}, v_{2}, v_{3}, v_{4}\right)=(2,1,3,4)$ constitutes a fair system of bets for 
the "transposed" problem $\left\langle N, A^{T}\right\rangle$, namely it solves

$$
\sum_{j \in N} a_{j i} v_{j}=\sum_{j \in N} a_{i j} v_{i} \quad i \in N
$$

we have that $2 \succeq_{R}^{d f b} \quad 1 \succeq_{R}^{d f b} \quad 3 \succeq_{R}^{d f b} \quad 4$ is the ranking of the players according to the dual fair-bets method.

Note that the fair-bets method seems to give more weight to wins against better players than to losses against worse players. The dual fair-bets method, on the other hand, seems to do just the opposite. Consider the above tournament. According to the fair-bets method, player 4's win against player 1 has more weight than his loss against player 3, and player 1 's loss to player 4 has less impact than his win against player 2. According to the dual fair-bets method, on the other hand, player 4's win against player 1 has less weight than his loss against player 3, and player 1's loss to player 4 has more impact than his win against player 2 .

\section{A characterization of the fair-bets method}

We now present some properties that we would expect methods to satisfy. The first three properties relate players that belong to different leagues and will allow us to extend a method defined on irreducible problems to a larger class of problems. The first two properties ensure that methods respect the "higher than" ordering of the leagues. The axioms are stated for an arbitrary method $\succeq$.

Dominance: For every problem $R=\langle N, A\rangle \in \mathcal{A}$, and for all players $i, j \in N$,

$$
[i] \mapsto[j] \text { implies } i \succ_{R} j \text {. }
$$

This property says that if one league is higher than another one, then all players in the former should be ranked strictly above all the players in the latter.

Another basic property that we require is the following. 
Quasi-completeness: For all problems $R=\langle N, A\rangle \in \mathcal{A}$ and for all players $i, j \in N$,

$$
i \nrightarrow j \text { and } j \nrightarrow i \text {, if and only if, } i \perp_{R} j \text {. }
$$

This property says that two players are incomparable if, and only if, neither of them beat the other one indirectly. In order for two players to be comparable there must be some grounds for indirect comparison, and, moreover, if at least one player indirectly beat the other, then the method must rank them.

Given the above two axioms, any two players belonging to different leagues will be either ranked one above the other, according to the relation between their respective leagues, or be incomparable if their leagues are. The only aspect that may distinguish among methods that satisfy the above two properties is the way they rank players within the same league. Before we state the next property we need some notation.

Given a ranking $\succeq_{R}$ of the players in $N$, we denote by $\succeq_{R} \mid[i]$ the restriction of $\succeq_{R}$ to the players in $[i]$. The next requirement imposes some consistency on the method. Specifically, the ranking of players within one league should be independent of the matches played by members of the other leagues.

Separability: For all problems $R=\langle N, A\rangle$ and for all players $i \in N, \succeq_{R} \mid[i]=\succeq_{R_{[i]}}$.

Note that this axiom relates problems with different sets of players.

Dominance and quasi-completeness allow us to determine the ranking of players belonging to different leagues. Separability implies that in order to rank two players in the same league, it is sufficient to focus on that league.

Once we adopt the three axioms discussed above, we only need to focus on irreducible problems. Formally, given a method $\succeq: \mathcal{A}_{I} \rightarrow \cup_{N \subseteq \mathbb{N}} \mathcal{R}_{N}$ defined on the class of irreducible problems, there is a unique extension, $\widehat{\succeq}$, of $\succeq$ to the class of all problems $\mathcal{A}$ that satisfies dominance, quasi-completeness, and separability. This extension is defined as follows. For all $R=\langle N, A\rangle \in \mathcal{A}$, and all $i, j \in N$

$$
\begin{aligned}
& \text { if }[i]=[j], \text { then } i \widehat{\succeq}_{R} j \Leftrightarrow i \succeq_{R_{[i]}} j \\
& \text { if }[i] \mapsto[j], \text { then } i \widehat{\succeq}_{R} j \\
& \text { if }[j] \mapsto[i], \text { then } j \widehat{\succeq}_{R} i
\end{aligned}
$$




$$
\text { otherwise, } \quad i \widehat{\perp}_{R} j \text {. }
$$

This method is the only one that satisfies dominance, quasi-completeness and separability, and coincides with $\succeq$ over the class of irreducible problems. We refer to this method as the natural extension of $\succeq$, and, with some abuse of notation, denote it by $\succeq$.

As a result of the above discussion we can define the fair-bets method to be the natural extension of the method $\succeq^{f b}$ defined in Example 1. Similarly, we define the dual fairbets method to be the natural extension of the method $\succeq^{d f b}$ defined in Example 2. Since they are natural extensions, these methods satisfy dominance, quasi-completeness, and separability.

The following axioms refer to problems with a fixed set of players, and will allow us to pin down a unique way to rank players in the same league.

Given a problem $R=\langle N, A\rangle \in \mathcal{A}$ and a permutation $\sigma: N \rightarrow N$, define the matrix $\sigma A$ by $(\sigma A)_{i j}=A_{\sigma(i), \sigma(j)}$, and the problem $\sigma R$ by $\sigma R=\langle N, \sigma A\rangle$.

Anonymity. For all $R=\langle N, A\rangle \in \mathcal{A}$ and all permutations $\sigma: N \rightarrow N, \sigma(i) \succeq_{R} \sigma(j)$ implies $i \succeq_{\sigma R} j$.

Anonymity is a standard property that requires that the method be independent of the names of the players.

The next axiom deals with the question of how to aggregate two problems with the same set of participants.

Quasi-flatness Preservation. Let $R_{1}=\langle N, A\rangle$ and $R_{2}=\langle N, B\rangle$ be two problems and let $R=\langle N, A+B\rangle$ be the combination of the two. Assume that $\succeq_{R_{1}}$ is quasi-flat. Then $\succeq_{R_{2}}$ is quasi-flat if and only if $\succeq_{R}$ is quasi-flat.

Quasi-flatness preservation requires that if two problems are such that the method cannot rank any player above another, then the method should still be unable to rank one player above another when consolidating the two problems into a single problem. And conversely, if two problems are such that in the first one the method does not rank one player above another, but in the second problem there are two players that are strictly ordered, then, when considering the two problems together, there should be at least two players who are ranked one above the other.

Note that quasi-flatness preservation implies the following property: 
Weak Homogeneity. For all problems $R=\langle N, A\rangle$ and all integers $k>0$, if $\succeq_{R}$ is quasi-flat, then so is $\succeq_{k R}$, where $k R=\langle N, k A\rangle$.

The last axiom requires that in some particular cases, increasing proportionally all the losses of one player should have a negative effect on his ranking.

Negative Responsiveness to Losses. Let $R=\langle N, A\rangle \in \mathcal{A}_{I}$ be an irreducible problem such that $\succeq_{R}$ is flat, and let $\Lambda=\operatorname{diag}\left(\lambda_{i}\right)_{i \in N}$ be a diagonal matrix such that $\left(\lambda_{i}\right)_{i \in N} \gg 0$, and $\langle N, A \Lambda\rangle \in \mathcal{A}$. Then $i \succeq_{\langle N, A \Lambda\rangle} j$ if and only if $\lambda_{i} \leq \lambda_{j}$.

Negative responsiveness to losses requires that if all players are equally ranked in some irreducible problem, and a new problem is obtained by multiplying each player's losses by some positive constant (which may be different for each player), then the players should be ranked in the new problem in a way that is inversely related to these constants: the larger the constant by which a player's losses have been multiplied, the lower his new rank. To motivate this requirement, let $f$ be a method, $\langle N, A\rangle$ an irreducible problem, and $\left\langle N, A^{\prime}\right\rangle$ a problem obtained from $\langle N, A\rangle$ by multiplying the $i$-th column of $A$ by an integer $\lambda_{i}>1$. Denote $f(N, A)$ and $f\left(N, A^{\prime}\right)$ by $\succeq$ and $\succeq^{\prime}$ respectively. Since an increase in player $i$ 's losses should negatively impact his standing more any other player's, we should have that if $j \succeq i$ then, $j \succeq^{\prime} i$ for all $j$. Indeed, the increase in $i$ 's losses should negatively impact player $i$ 's standing more than any other player's. The question is how the relative ranking of the players other than $i$ should be affected by the above modification. Note first that neither player $i$ 's vector of wins nor the distribution of his losses has changed. Therefore, if one takes the view that player $i$ 's ranking derives from his victories over the other players, and that the value of those victories should be indirectly distributed among the other players proportionally to their victories over $i$, it would be reasonable to require that the relative ranking of any two players other than $i$ not change by the above modification. That is, the value of player $i$ 's victories, whose number has not changed, is distributed among the other players in the same proportions as before. The axiom of negative responsiveness to losses is an expression of the above idea when iterated several times. If $f$ ranks all players equally in some problem $(N, A)$, and if player $j$ 's column in $A$ is multiplied by some integer $\lambda_{j} \geq 1$, for all players $j \in N$, then the new ranking should be inversely related to the value of the $\lambda_{j}$ s. The rationale is that multiplying $j$ 's losses affects only his relative ranking vis a vis the rest of the players, and not the other players' ranking relative to each other. 
We can now state the main result of the paper.

Theorem 1 The fair-bets method $\succeq^{f b}$ is the unique method that satisfies dominance, quasi-completeness, separability, anonymity, quasi-flatness preservation and negative responsiveness to losses.

Before we prove the theorem, we describe the idea behind the proof of the uniqueness part. Consider an irreducible problem $\langle N, A\rangle$. As we know, the fair-bets method ranks the players in $N$ according to a positive eigenvector, $v$, of the matrix $C_{A}^{-1} A$. That is, $v$ solves the system of equations

$$
\sum_{j \in N} a_{i j} v_{j}=\sum_{j \in N} a_{j i} v_{i} \quad i \in N .
$$

This means that the matrix $A \operatorname{diag}\left(v_{i}\right)_{i \in N}$, whose typical entry is $a_{i j} v_{j}$, is balanced.

If the players in the problem $\left\langle N, A \operatorname{diag}\left(v_{i}\right)_{i \in N}\right\rangle$ were equally ranked, it would follow from negative responsiveness to losses that the players in the original problem are ranked according to $v$. Hence, the strategy of the proof is to show that a method that satisfies all the axioms ranks all players equally in every balanced problem. The proof is in two steps. First, we prove it for strongly balanced problems and then, using quasi-flatness preservation, we extend it to all balanced problems. For strongly balanced problems, we apply Birkhoff's theorem (Birkhoff (1946), see also Minc (1988)), according to which, every doubly stochastic matrix can be written as a convex combination of permutation matrices. This immediately implies that every strongly balanced matrix can be written as a linear combination of permutation matrices with positive coefficients. Using quasi-completeness and anonymity, we can prove that the method must rank all players equally in every problem $\langle N, P\rangle$, where $P$ is a permutation matrix. Finally, quasi-flatness preservation implies that the same is true for any combination of permutation matrices.

We now turn to the formal proof of the theorem.

Proof : We will first argue that the fair-bets method satisfies all the axioms. Since the fair-bets method is the natural extension of a method defined on the class of irreducible problems, it automatically satisfies dominance, quasi-completeness and separability. It should be clear that it also satisfies anonymity. 
Step 1 The fair-bets method satisfies quasi-flatness preservation.

Let $R_{1}=\langle N, A\rangle$ be a problem such that $\succeq_{R_{1}}^{f b}$ is quasi-flat. Since the fair-bets method is the natural extension of a method defined on the class of irreducible problems, all the leagues in $R_{1}$ are incomparable. Further, for all $i \in N$, quasi-flatness of $\succeq_{R_{1}}^{f b}$ means that

$$
\sum_{j \in[i]} a_{i j}=\sum_{j \in[i]} a_{j i}
$$

which implies that all leagues are balanced. By Lemma $1, R_{1}$ is balanced.

Now consider $R_{2}=\langle N, B\rangle \in \mathcal{A}$ and $R=\langle N, A+B\rangle$. We have to show that $\succeq_{R_{2}}^{f b}$ is quasi-flat if and only if $\succeq_{R}^{f b}$ is quasi-flat. If $\succeq_{R_{2}}^{f b}$ is quasi-flat, then by the above reasoning $R_{2}$ is balanced. Therefore, $R$, as a sum of two balanced problems, is balanced too. By Lemma 1, all its leagues are balanced and incomparable. By definition of the fair-bets method, $\succeq_{R}^{f b}$ is quasi-flat. As for the other direction, if $\succeq_{R}^{f b}$ is quasi-flat, then by the same reasoning as in the first paragraph of the proof, $R$ is balanced. Since $R_{1}$ is also balanced, we conclude that $R_{2}$ is balanced as well. By Lemma 1 , all its leagues are incomparable and balanced, implying that $\succeq_{R_{2}}^{f b}$ is quasi-flat.

Step 2 The fair-bets method satisfies negative responsiveness to losses.

Let $R=\langle N, A\rangle$ be an irreducible problem, and assume that $\succeq_{R}^{f b}$ is flat. This means that $\sum_{j \in N} a_{i j}=\sum_{j \in N} a_{j i}$ for all $i \in N$. Let $\left(\lambda_{i}\right)_{i \in N} \gg 0$ be a vector of positive entries such that $\langle N, A \Lambda\rangle \in \mathcal{A}$, where $\Lambda=\operatorname{diag}\left(\lambda_{i}\right)_{i \in N}$. Then for all $i \in N$

$$
\sum_{j \in N}\left(\lambda_{j} a_{i j}\right) \frac{1}{\lambda_{j}}=\sum_{j \in N}\left(\lambda_{i} a_{j i}\right) \frac{1}{\lambda_{i}}
$$

This means that $\left(\lambda_{i}^{-1}\right)_{i \in N}$ is an eigenvector of $C_{A \Lambda}^{-1} A \Lambda$ and thus the fair-bets method ranks the players in $\langle N, A \Lambda\rangle$ according to $\left(\lambda_{i}^{-1}\right)_{i \in N}$

We now show that any method that satisfies the six axioms, coincides with the fair-bets method. By dominance, separability and quasi-completeness, it is enough to show that the statement holds for all irreducible problems. Let $N$ be a set of players. In what follows we restrict attention to problems with player set $N$. Therefore we omit $N$ from the notation, and we denote a problem by its matrix. 
Step 3 Let $\succeq$ be a method that satisfies anonymity and quasi-completeness. If $P$ is a permutation matrix with $p_{i i}=0$ for all $i$, then $\succeq_{P}$ is quasi-flat.

Since $P$ is a permutation matrix, the players of each league form a cycle. By anonymity and quasi-completeness, all players in each cycle are equally ranked. Further, $P$ 's leagues are incomparable, and hence, by quasi-completeness, $\succeq_{P}$ is quasi-flat.

Step 4 If $\succeq$ satisfies anonymity, quasi-completeness, quasi-flatness preservation and negative responsiveness to losses, then for any strongly balanced and irreducible problem A, $\succeq_{A}$ is flat.

Let $A$ be a strongly balanced and irreducible problem. Since $A$ is strongly balanced, by Birkhoff's theorem (Birkhoff (1946)), A can be written as

$$
A=\sum_{t=1}^{T} k_{t} P_{t}
$$

where for each $t, k_{t}$ is a positive constant and $P_{t}$ is a permutation matrix. Since $A$ is a matrix with integer entries, all the $k_{t}$ can be chosen to be rational numbers. Let $\kappa \in \mathbb{N}$ be such that $\kappa k_{t} \in \mathbb{N}$ for $t=1, \ldots T$. Then, denoting $\kappa_{t}=\kappa k_{t}$, we have

$$
\kappa A=\sum_{t=1}^{T} \kappa_{t} P_{t} .
$$

By Step $3, \succeq_{P_{t}}$ is quasi-flat. By weak homogeneity, $\succeq_{\kappa_{t} P_{t}}$ is quasi-flat for $t=1, \ldots, T$. By quasi-flatness preservation, $\succeq_{\kappa A}$ is also quasi-flat. Since $A$ is irreducible, so is $\kappa A$ and consequently $\succeq_{\kappa A}$ is flat. By negative responsiveness to losses, $\succeq_{A}$ is flat.

Step 5 If $\succeq$ satisfies anonymity, quasi-completeness, quasi-flatness preservation and negative responsiveness to losses, then for any balanced and irreducible problem $A, \succeq_{A}$ is flat.

Let $A$ be an irreducible balanced problem and let $b=2 \max _{i j} a_{i j}$. Consider the problem $2 A+b J$, where $J$ is the "unit matrix", whose off-diagonal entries are all 1 and whose diagonal entries are 0 . By anonymity, $\succeq_{J}$ is flat, and by weak homogeneity so is $\succeq_{b} J$. By quasi-flatness preservation, it is enough to show that $\succeq_{2 A+b J}$ is flat. Let $B=\left(b_{i j}\right)$ be 
the problem defined by

$$
b_{i j}= \begin{cases}0 & \text { if } i=j \\ b-\left(a_{i j}+a_{j i}\right) & \text { otherwise }\end{cases}
$$

Note that $2 A+b J=(2 A+B)+(b J-B)$. The matrix $2 A+B$ is a strongly balanced problem. Indeed, since both $A$ and $B$ are balanced, so is $2 A+B$. Further, for all $i \in N$

$$
\begin{aligned}
\sum_{j \neq i}\left(2 a_{i j}+b_{i j}\right) & =\sum_{j \neq i} 2 a_{i j}+\sum_{j \neq i}\left(b-a_{i j}-a_{j i}\right) \\
& =\sum_{j \neq i} 2 a_{i j}+(n-1) b-\sum_{j \neq i} 2 a_{i j} \\
& =(n-1) b,
\end{aligned}
$$

which implies that $2 A+B$ is strongly balanced. Therefore, by Step $4, \succeq_{2 A+B}$ is flat. Further $b J-B$ is a symmetric problem, therefore, by quasi-flatness preservation, anonymity, and quasi-completeness, $\succeq_{b J-B}$ is quasi-flat. Then, by quasi-flatness preservation $\succeq_{(2 A+B)+(b J-B)}$ is quasi-flat. Since $2 A+b J=(2 A+B)+(b J-B)$ is irreducible, $\succeq_{2 A+b J}$ is flat.

Step 6 Let $\succeq$ be a method that satisfies anonymity, quasi-completeness, quasi-flatness preservation, and negative responsiveness to losses. Then, for all irreducible problems A, $\succeq_{A}=\succeq_{A}^{f b}$.

Let $A$ be an irreducible problem and let $v=\left(v_{i}\right)_{i \in N}$ be a positive, integer-valued, eigenvector of $C_{A}^{-1} A$. That is, $v$ satisfies

$$
\sum_{j \in N} a_{i j} v_{j}=\sum_{j \in N} a_{j i} v_{i} \quad i \in N
$$

Then $A^{\prime}=A \operatorname{diag}\left(v_{i}\right)_{i \in N}$ is a balanced and irreducible problem. By Step $5, \succeq_{A^{\prime}}$ is flat. Since $A=A^{\prime}\left(\operatorname{diag}\left(v_{i}\right)_{i \in N}\right)^{-1}$, by negative responsiveness to losses,

$$
i \succeq_{A} j \Leftrightarrow 1 / v_{i} \leq 1 / v_{j} \Leftrightarrow v_{i} \geq v_{j} \Leftrightarrow i \succeq_{A}^{f b} j
$$

which proves the required equality. 
Step 6 completes the proof of the theorem.

Remark 1 If we replace the axiom of negative responsiveness to losses with a dual one of positive responsiveness to wins, which is defined in an analogous and obvious way, a similar proof would give a characterization of the dual fair-bets method. This shows also that there is no method that satisfies both negative responsiveness to losses and positive responsiveness to wins together with the rest of our axioms, except, of course, within the class of two-player problems where the fair-bets and the dual fair bets methods coincide.

Remark 2 The axiom of dominance is used only to determine the relative ranking between any two players who belong to two different and comparable leagues. We could dispense with this axiom if we restricted our attention to the subclass of problems that consist of only one league or of several incomparable leagues. The same proof of Theorem 1 shows that the fair-bets method is the only method for this smaller class of problems that satisfies quasi-completeness, separability, anonymity, quasi-flatness preservation and negative responsiveness to losses.

\section{Independence of the axioms}

In this section we briefly consider the question of independence of the axioms used in this paper. We list below, along with each axiom, an example of a method that satisfies all the axioms mentioned in Theorem 1 except for the one under consideration. In each case we indicate in boldface which axiom fails.

Dominance The method $\succeq$ such that for all $R=\langle N, A\rangle, i, j \in N$,

- if $[i] \mapsto[j]$ then $j \succeq_{R} i$

- otherwise $i \succeq_{R} j$ if and only if $i \succeq_{R}^{f b} j$. 
Quasi-completeness The method that coincides with the fair-bets except for problems with exactly two incomparable and strongly balanced leagues. In this case, the method ranks all players equally.

Separability Consider the method that ranks the players in a problem in the following way. If the problem has only one league, then players are ranked according to the fair-bets method. Otherwise, the players are ranked according to the dual fair-bets. To see that this method satisfies negative responsiveness to losses, note that this axiom applies only to irreducible problems and in this class the method coincides with the fair-bets, which satisfies the axiom. To see that this method satisfies quasiflatness preservation, note that it yields a quasi-flat ranking if and only if the problem is balanced.

Anonymity Fix an arbitrary but complete (strict) order $\tilde{\succ}$ of the players, and consider the natural extension of the method (defined on the class of irreducible problems) that ranks the players of a league according to $\tilde{\succ}$.

Negative responsiveness to losses The natural extension of the method that ranks all players equally in all irreducible problems. Another example is the dual fair-bets method.

Quasi-flatness Preservation The natural extension of the method, defined on the class of irreducible problems, that ranks the players according to the reciprocal of their total number of losses. To see that this method does not satisfy quasi-flatness preservation, consider the problems defined by the following matrices:

$$
A=\left(\begin{array}{llll}
0 & 1 & 0 & 0 \\
1 & 0 & 0 & 0 \\
0 & 0 & 0 & 1 \\
0 & 0 & 1 & 0
\end{array}\right), B=\left(\begin{array}{llll}
0 & 0 & 0 & 2 \\
0 & 0 & 3 & 0 \\
0 & 3 & 0 & 0 \\
2 & 0 & 0 & 0
\end{array}\right), A+B=\left(\begin{array}{llll}
0 & 1 & 0 & 2 \\
1 & 0 & 3 & 0 \\
0 & 3 & 0 & 1 \\
2 & 0 & 1 & 0
\end{array}\right)
$$

It is clear that the method results in quasi-flat rankings for both $A$ and $B$ but not for $A+B$. 


\section{Appendix}

Proof of Lemma 1: The "if" part is simple. If all leagues are incomparable, then for all $i \in N$ and for all $j \notin[i], a_{i j}=a_{j i}=0$. If, in addition, the league $[i]$ is balanced, then

$$
\sum_{j \in N} a_{i j}=\sum_{j \in[i]} a_{i j}=\sum_{j \in[i]} a_{j i}=\sum_{j \in N} a_{j i} \quad \forall i \in N
$$

For the "only if" part, assume $R$ is balanced. Since the "higher than" relation between leagues is irreflexive and transitive, we can find a league, say $[k]$, that is maximal with respect to that relation. That is, no player outside $[k]$ beat a player in $[k]$. Formally, $a_{j i}=0$ for all $i \in[k]$ and for all $j \notin[k]$. This means that

$$
\sum_{j \in N} a_{j i}=\sum_{j \in[k]} a_{j i} \quad \forall i \in[k] .
$$

We will show that $[k]$ is also a minimal league. That is, that $a_{i j}=0$ for all $i \in[k]$ and for all $j \notin[k]$. This implies that

$$
\sum_{j \in[k]} a_{i j}=\sum_{j \in N} a_{i j} \quad \forall i \in[k]
$$

which together with equation (2) and the fact that $R$ is balanced means that $[k]$ is balanced.

In order to show that $[k]$ is minimal, note that since $R$ is balanced and $[k]$ is maximal,

$$
\sum_{j \in N} a_{i j}=\sum_{j \in N} a_{j i}=\sum_{j \in[k]} a_{j i} \quad \forall i \in[k] .
$$

Adding over all $i \in[k]$, we get

$$
\sum_{i \in[k]} \sum_{j \in N} a_{i j}=\sum_{i \in[k]} \sum_{j \in[k]} a_{j i}
$$

or, splitting the sum on the left-hand side,

$$
\sum_{i \in[k]} \sum_{j \notin[k]} a_{i j}+\sum_{i \in[k]} \sum_{j \in[k]} a_{i j}=\sum_{i \in[k]} \sum_{j \in[k]} a_{j i} .
$$


Since $\sum_{i \in[k]} \sum_{j \in[k]} a_{i j}=\sum_{i \in[k]} \sum_{j \in[k]} a_{j i}$, we have that $\sum_{i \in[k]} \sum_{j \notin[k]} a_{i j}=0$, which implies that $a_{i j}=0$ for all $i \in[k]$ and $j \notin[k]$. In other words, $[k]$ is a minimal league. Further, since $[k]$ is an arbitrary maximal league, we conclude that all maximal leagues are also minimal, implying that all leagues are incomparable.

\section{References}

Arrow, K. J. (1963). Social Choice and Individual Values (2nd. ed.). New Haven: Yale University Press.

Birkhoff, G. (1946). Tres observaciones sobre el álgebra lineal. Univ. Nac. Tucumán Rev. Ser. A 5, 147-150.

Bouyssou, D. (1992). Ranking methods based on valued preference relations: A characterization of the net flow method. European Journal of Operational Research 60, $61-67$.

Conner, G. R. and C. P. Grant (2000). An extension of Zermelo's model for ranking by paired comparisons. European Journal of Applied Mathematics 11, 225-247.

Daniels, H. E. (1969). Round-robin tournament scores. Biometrika 56, 295-299.

David, H. A. (1987). Ranking from unbalanced paired-comparison data. Biometrika 74, $432-436$.

David, H. A. (1988). The Method of Paired Comparisons (2 ed.). London: Charles Griffin and Company.

Henriet, D. (1985). The Copeland choice function: an axiomatic characterization. Social Choice and Welfare 2, 49-63.

Herings, J.-J., G. van der Laan, and D. Talman (2004). The positional power of nodes in digraphs. Social Choice and Welfare, forthcoming.

Kano, M. and A. Sakamoto (1985). Ranking the vertices of a paired comparison digraph. SIAM Journal on Algebraic and Discrete Methods 6, 79-92.

Keener, J. P. (1993). The Perron-Frobenius theorem and the ranking of footbal teams. SIAM Review 35, 80-93. 
Kendall, M. G. (1955). Further contributions to the theory of paired comparisons. Biometrics 11, 43-62.

Laslier, J. (1997). Tournament Solutions and Majority Voting. Berlin: Springer.

Levchenkov, V. S. (1992). Social choice theory: a new insight. Discussion paper, Institute of Systems Analysis, Moscow.

Levchenkov, V. S. (1995). Self-consistent rule for group choice. I: Axiomatic approach. Discussion Paper 95-3, Conservatoire National des Arts et Métiers.

Minc, H. (1988). Nonnegative Matrices. John Wiley \& Sons.

Moon, J. W. (1968). Topics on Tournaments. New York: Holt, Rinehart and Winston.

Moon, J. W. and N. J. Pullman (1970). On generalized tournament matrices. SIAM Review 12, 384-399.

Moulin, H. (1988). Axioms of Cooperative Decision Making. Cambridge, U. K.: Cambridge University Press.

Palacios-Huerta, I. and O. Volij (2004). The measurement of intellectual influence. Econometrica 72, 963-977.

Rubinstein, A. (1980). Ranking the participants in a tournament. SIAM Journal of Applied Mathematics 38, 108-111.

Sen, A. (1970). Individual Choice and Social Welfare. San Francisco: Holden Day.

Slutzki, G. and O. Volij (2004). Scoring of web pages and tournaments - axiomatizations. Social Choice and Welfare, forthcoming.

van den Brink and R. Gilles (2000). Measuring domination in directed networks. Social Networks 22, 141-157.

Wei, T. (1952). The algebraic foundation of ranking theory. Ph. D. Thesis, Cambridge University.

Zermelo, E. (1929). Die Berechnung der Turnier-Ergebnisse als ein Maximalproblem der Wahrscheinlichkeitsrechnung. Mathematische Zeitschrift 29, 436-460. 